\title{
A NOTE ON TOPOLOGICAL PROPERTIES OF NORMED LINEAR SPACES ${ }^{1}$
}

\author{
V. L. KLEE, JR.
}

In a recent paper [2], the author established certain topological properties of infinite-dimensional normed linear spaces. A fundamental tool was the theorem of Smulian [4] to the effect that every nonreflexive normed linear space contains a decreasing sequence of nonempty bounded closed convex sets whose intersection is empty. Thus several of the main results were obtained only for nonreflexive spaces and, using Mazur's homeomorphism [3] between $L^{1}$ and $L^{p}$, for infinite-dimensional $L^{p}$ spaces. In the present note we produce an alternative tool, dealing with sequences of linearly bounded closed convex sets, whereby some of the results of [2] can be established for arbitrary infinite-dimensional normed linear spaces.

A subset of a linear space is linearly bounded provided it intersects each line in a bounded set. Our substitute for Smulian's theorem is the following:

THEOREM 1. Every infinite-dimensional normed linear space $E$ contains a decreasing sequence $C_{\alpha}$ of unbounded but linearly bounded closed convex sets whose intersection is empty.

Proof. We assume without loss of generality that $E$ is separable, and hence that $E$ admits a sequence $f_{\alpha}$ of continuous linear functionals such that $\bigcap_{1}^{\infty} f_{i}^{-1} 0=\{\phi\}$. We describe first the construction of $C_{0}$.

For each $i$ and $j$ let $Q_{i}^{j}=f_{i}^{-1}[-j, j]$. Let $n_{1}=1$ and choose $p_{1} \in Q_{1}^{n_{1}}$ such that $\left\|p_{1}\right\|=1$. Having selected $n_{i}$ and $p_{i}$ for $1 \leqq i<k$, choose $n_{k}$ so that $\left\{p_{i} \mid 1 \leqq i<k\right\} \subset Q_{k}^{n_{k}}$ and then choose $p_{k} \in \bigcap_{1}^{\mathbf{k}} Q_{i}^{n_{i}}$ so that $\left\|p_{k}\right\|=k$. Proceeding by mathematical induction, we obtain infinite sequences $n_{\alpha}$ of positive integers and $p_{\alpha}$ of points of $E$. Let $C_{0}=\bigcap_{1}^{\infty} Q_{i}^{n_{i}}$. Then clearly $C_{0}$ is closed and convex; and $\left\{p_{i} \mid 1 \leqq i<\infty\right\} \subset C_{0}$, so $C_{0}$ is unbounded. Now consider an arbitrary point $x \in E \sim\{\phi\}$. For some $k, f_{k} x=\delta \neq 0$, and then $C_{0} \cap(]-\infty, \infty[x) \subset|\delta|^{-1} n_{k}[-1,1] x$. Thus $C_{0}$ contains no ray through $\phi$, and since $C_{0}$ is closed and convex it follows easily that $C_{0}$ is linearly bounded.

To complete the construction of $C_{\alpha}$, we observe that since $C_{0}$ is unbounded, then by the uniform boundedness principle [1], $E$ admits a continuous linear functional $f$ such that $\sup _{c_{0}} f=\infty$. Thus with

Received by the editors February 9, 1955.

${ }^{1}$ Sponsored by the Office of Ordnance Research, U. S. Army, under Contract DA-04-200-ORD-292. 
$C_{i}=C_{0} \cap f^{-1}[i, \infty]$ for each $i, C_{\alpha}$ is the desired sequence of sets. Theorem 1 has been proved.

Now using Theorem 1 in lieu of Smulian's theorem to obtain a result similar to (I 4.1) of [2], and following the arguments of \$II 1 of [2], it is possible to prove the following:

Theorem 2. Suppose $E$ is an infinite-dimensional normed linear space, $Y$ is a compact subset of $E, U$ is the unit cell $\{x \mid\|x\| \leqq 1\}$ of $E$, $S$ is the unit sphere $\{x \mid\|x\|=1\}$ of $E, H$ is a hyperplane in $E$, and $Q$ is a closed half-space bounded by $H$. Then

(i) $E \sim Y$ is homeomorphic with $E$.

(ii) There is a homeomorphism of $E \sim$ Int $U$ onto $U$ which is the identity on $S$.

(iii) There is a homeomorphism of period two without fixed points of $E$ onto $E$ which takes $U$ onto $U$.

(iv) There is a homeomorphism of $Q$ onto $U$ which takes $H$ onto $S$.

It is not clear that Theorem 1 will help to extend the Isotopy Theorem of [2] to more general spaces, for considerations of boundedness enter at several points in the proof of the latter.

\section{REFERENCES}

1. Stefan Banach, Théorie des opérations linéaires, Warsaw, 1932.

2. V. L. Klee, Jr., Convex bodies and periodic homemorphisms in Hilbert space, Trans. Amer. Math. Soc. vol. 74 (1953) pp. 10-43.

3. S. Mazur, Une remarque sur l'homeomorphie des champs fonctionnels, Studia Math. vol. 1 (1929) pp. 83-85.

4. V. Smulian, On the principle of inclusion in the space of the type (B), Rec. Math. (Mat. Sbornik) N. S. vol. 5 (1939) pp. 317-328.

UNIVERSITY OF WASHINGTON 